\title{
The Consequences of Not Looking at the Eosinophil Count: Pulmonary Infiltrates and Eosinophilia Syndrome (PIE) Probably Caused by Ciprofloxacin
}

\author{
Martina Del Torre, Cristiano Vitale, Donato Caliandro \\ Clinica Medica, Department of Experimental and Clinical Medical Sciences (DISM), \\ Hospital "Santa Maria della Misericordia", Udine, Italy
}

Received: $12 / 03 / 2015$

Accepted: 08/06/2015

Published: $23 / 06 / 2015$

How to cite this article: Del Torre M, Vitale C, Caliandro D. The consequences of not looking at the eosinophil count: pulmonary infiltrates and eosinophilia syndrome (PIE) probably caused by ciprofloxacin. EJCRIM 2015;2:doi: 10.12890/2015_000201

Conflicts of Interests: The authors declare that they have no conflicts of interest in this research.

Aknowledgements: The authors declare they had patient's permission for reporting this case report.

This article is licensed under a Commons Attribution Non-Commercial 4.0 License

ABSTRACT

Objectives: To describe a case of pulmonary infiltrates and eosinophilia (PIE syndrome) probably caused by ciprofloxacin.

Materials and methods: A 64-year-old woman was admitted to our department with suspected hospital-acquired pneumonia and treated with antibiotics. She had no symptoms but had peripheral eosinophilia. She had recently been given ciprofloxacin for a urinary tract infection.

Results: The patient spontaneously improved after exhaustive negative investigations.

Conclusion: We concluded that this patient had PIE syndrome probably caused by ciprofloxacin.

\section{LEARNING POINTS}

- The eosinophil count is an important clinical parameter that is often overlooked.

- Löffler described migratory pulmonary infiltrates in patients with eosinophilia and few or no pulmonary symptoms.

- Drugs are commonly involved in the pathogenesis of simple pulmonary eosinophilia.

- As far as we know, this is the first case of pulmonary infiltrates and eosinophilia syndrome (PIE) caused by ciprofloxacin to be reported.

\section{KEYWORDS}

Ciprofloxacin, eosinophil count, pulmonary infiltrates.

\section{INTRODUCTION}

The eosinophil count is now provided as part of all routine full blood counts but is often overlooked by clinicians. This case demonstrates how failure to consider this simple test delayed recognition of a relatively rare, yet benign self-limiting condition. This resulted in the patient undergoing several invasive, unpleasant and expensive investigations and unnecessary treatments.

\section{CASE REPORT}

A 64-year-old woman presented to our emergency unit with altered mental status and hyperglycaemia. She had a past history of diabetes mellitus complicated by retinopathy, peripheral arteriopathy and neuropathy, and chronic kidney failure. She had chronic multifactorial anaemia and a long-standing creatinine value of $1.8 \mathrm{mg} / \mathrm{dl}$. She also had a history of allergy to penicillin.

The patient had been discharged 5 days previously from a medical unit with a diagnosis of 'toxidermic skin reaction of iatrogenic origin'. This had occurred a few days after she had been given ciprofloxacin for a urinary tract infection. At that time she had a normal white cell count, but eosinophils were $12.8 \%$ of the total $(798 / \mathrm{mmc})$.

A chest $x$-ray in the emergency department showed diffuse infiltrates throughout the right lung. Although the patient was afebrile and had no relevant symptoms, hospital-acquired pneumonia was suspected and treatment with meropenem was started. After 2 days her glycaemic control improved and she was transferred to our medical ward.

On admission to our ward she was afebrile, asymptomatic, and denied any fever, chills, cough or dyspnoea at any time over the previous few days. Physical examinations revealed bilateral crackles in her lower and middle lung fields. A papular itchy rash started to develop on her thighs and extended to her calves. Over the next few days inflammatory markers decreased, her C-reactive protein (CRP) was 48 mg/l and her white cell count remained normal, but her eosinophils increased to $1300 / \mathrm{mmc}$.

During her stay in hospital, a large number of investigations were performed to exclude multiple suspected aetiologies. IgE and cortisol levels were normal. Stool examination for parasites on three different days was negative. A CT scan was ordered because lung cancer was 


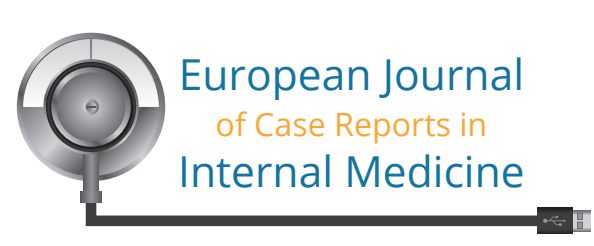

suspected. Prior to this examination, steroid treatment was prescribed to prevent a contrast reaction in view of the patient's previous allergic history.

The day after steroid treatment and the CT scan, her eosinophils were 0.0 and CRP was $22 \mathrm{mg} / \mathrm{l}$. However, her eosinophils started to increase again a few days later. Churg-Strauss syndrome was then suspected even though the patient had never had asthma. Her autoimmune panel was negative: rheumatoid factor, ANA, ENA and ANCA were all negative. A skin biopsy showed non-specific changes.

The CT scan showed bilateral 'ground glass' opacities, confined mainly to the upper lobes and the right parahilar region, and bilateral pleural effusions that were greater on the right side $\left(7 \mathrm{~cm}\right.$ versus $4 \mathrm{~cm}$ on the left side). Over a litre of clear yellow exudate that contained $219 \times 10^{3}$ leucocytes (4\% eosinophils) was aspirated on thoracentesis; cultures for aerobic and anaerobic organisms were negative; cytology showed inflammation. An abdominal scan was normal.

Shortly after thoracentesis, a chest $x$-ray showed complete disappearance of pulmonary infiltrates.

Chest physicians were consulted and performed a bronchoscopy and bronchoalveolar lavage. Both these investigations were normal. A transbronchial biopsy was then considered but not performed because by this time the pulmonary infiltrates had disappeared and patient's eosinophil count had returned to normal.

\section{DISCUSSION}

In most bacterial infections, eosinophils disappear from the blood, probably as a consequence of steroid production resulting from stress. The return of eosinophils in normal numbers, therefore, suggests the beginning of recovery.

This case highlights the importance of the eosinophil count. Eosinophils were named after Eos, the Greek goddess of dawn. In infections, eosinophilia is generally a favourable prognostic sign, and the reappearance of eosinophils has been called the dawn of recovery ${ }^{[1]}$. This patient was misdiagnosed in the emergency department as having hospital-acquired pneumonia, and even though she had no symptoms or fever, a broad-spectrum antibiotic was started. Her eosinophilia was not noticed in the emergency room, and then masked by steroids given before the CT scan to reduce the risk of a reaction to contrast. There then followed several further invasive, unpleasant and expensive investigations, before it became clear that the patient was recovering spontaneously and her chest x-ray was returning to normal.

The syndrome of pulmonary infiltrates and eosinophilia (PIE) described by Löffler is a transient illness with blood eosinophilia and radiographic shadowing. Symptoms are mild or absent and tend to resolve quickly after a few days ${ }^{[2]}$. Most cases are caused by parasites or drugs; laboratory tests might show mild blood eosinophilia. On bronchoalveolar lavage the eosinophil count may be elevated ${ }^{[3]}$. On imaging, most patients have peripheral densities, usually with an interstitial and alveolar pattern ${ }^{[4]}$. No treatment is required for this self-limiting condition. In severe cases, corticosteroids are effective.

Ciprofloxacin was the most likely cause of this case of PIE because it was the only new drug taken by the patient at the time of her presentation. Although Steiger et al. reported a case of ciprofloxacin-induced acute interstitial pneumonitis in a 68 -year-old female patient ${ }^{[5]}$, as far as we know this is the first case of PIE caused by ciprofloxacin to be reported.

\section{CONCLUSIONS}

We concluded with a diagnosis of ciprofloxacin-induced simple pulmonary eosinophilia because of the absence of symptoms, the transient pulmonary infiltrates, the self-limiting eosinophilia, the need for no therapy and the temporal relationship with ciprofloxacin use.

\section{REFERENCES}



